\title{
Therapeutic Recommendations for the Treatment of Toenail Onychomycosis in the US
}

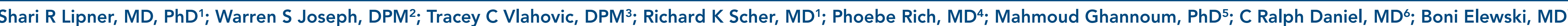

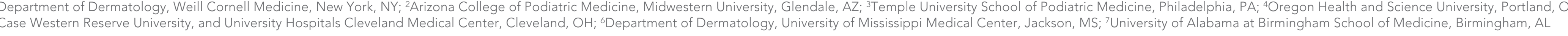

SYNOPSIS

- Onychomycosis - a fungal infection of the nail bed or plate-affects up to $14 \%$ of
individuals in North America',2

- It is undertreated and treatment is challenging as toenail growth can take up to 12 months
or more, the nail plate may prevent drug penentration, and disease recurrence is common

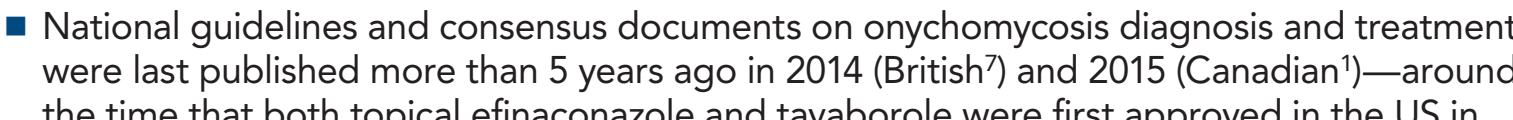
the time that both topical efinaconazole and tavaborole were first approved in the US in - Since then, more clinical data, post hoc analyses, meta-analysses, and FDA-approved
indications have become available for onychomycosis drugs - As such, updated medical guidance is needed

OBJECTIVE AND METHODS

- To provide recommendations for the diagnosis and therapeutic treatment of toenail

- Included here is a decision tree for choosing appropriate medications based on disease
severity and patient characcteristics, as well as an example handout intended for patients on RESULTS

Diagnosis, Testing, and Clinical Presentation

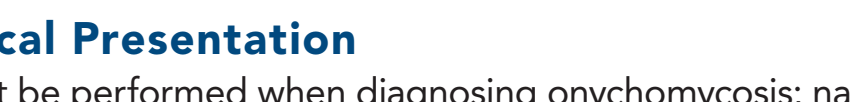
dystrophy can be induced by tother disorders, and many
mimic onychomycosis should be ruled out (Figure 1)

- Laboratory testing should also be performed to identify the infecting organism and
exclude non-fungal onditioss Figire 1 shows common options that are used in
conjunction with clinical diagnosis

FIGURE 1. Differential Diagnosis Decision Tree

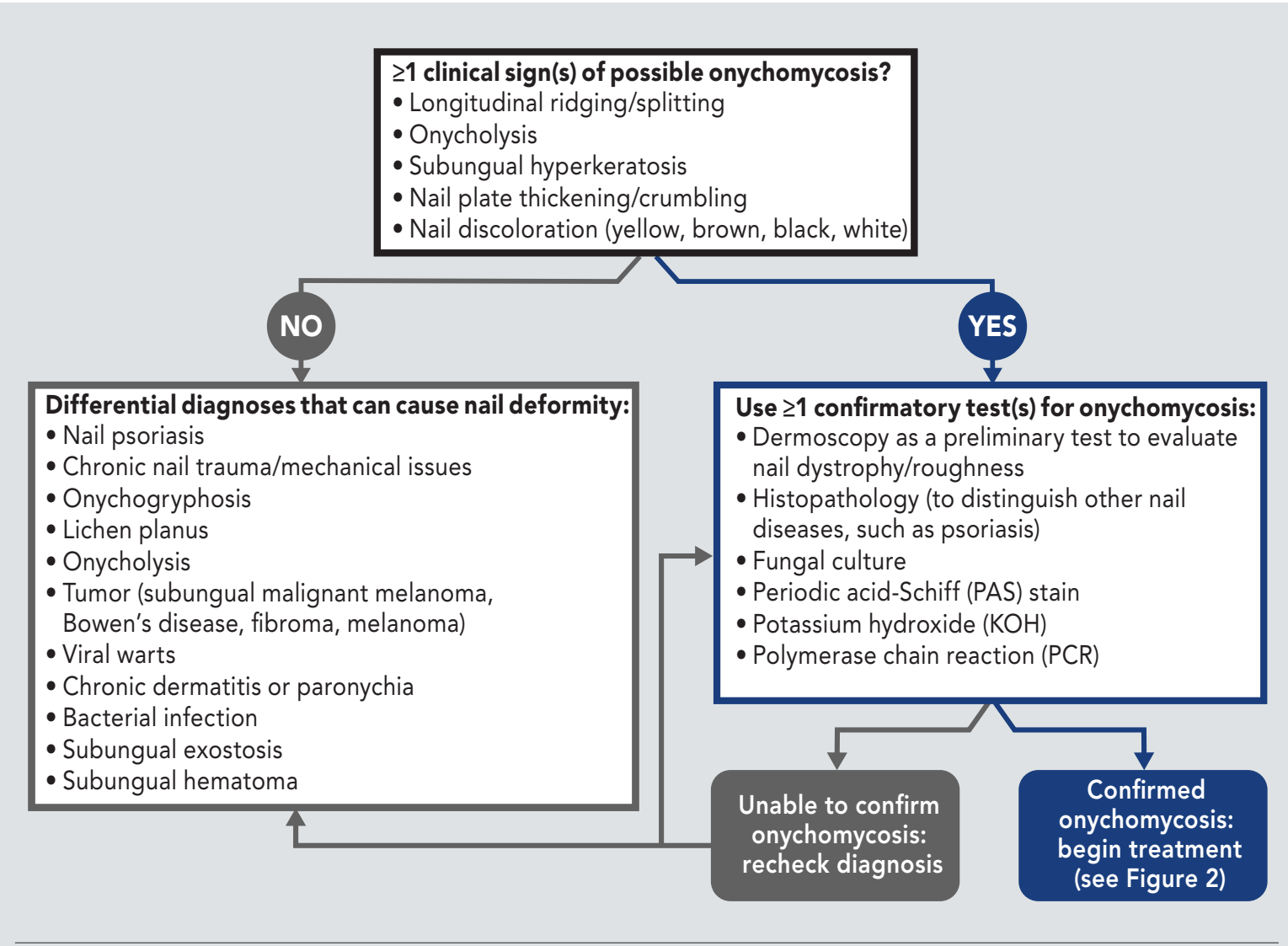

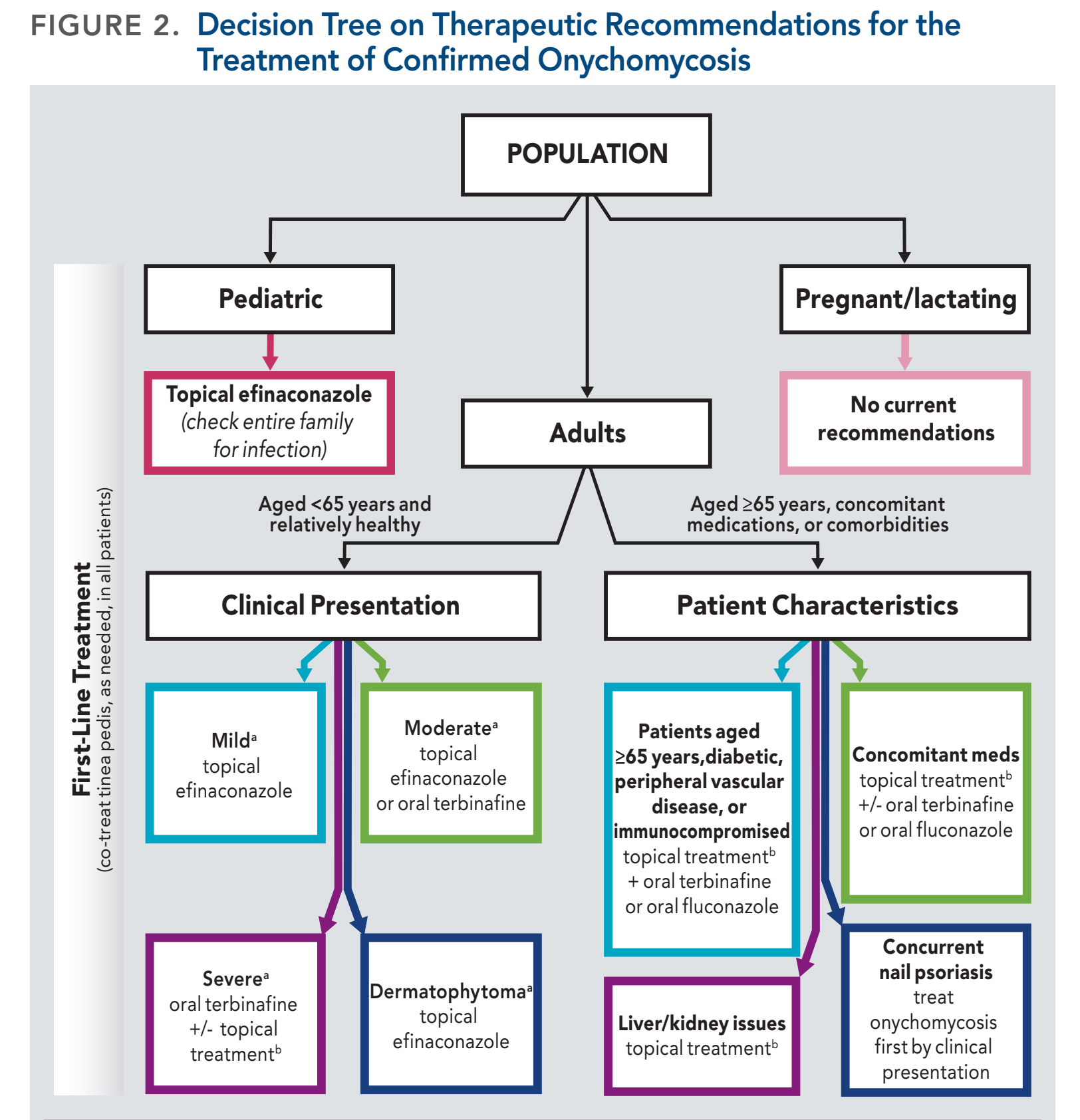

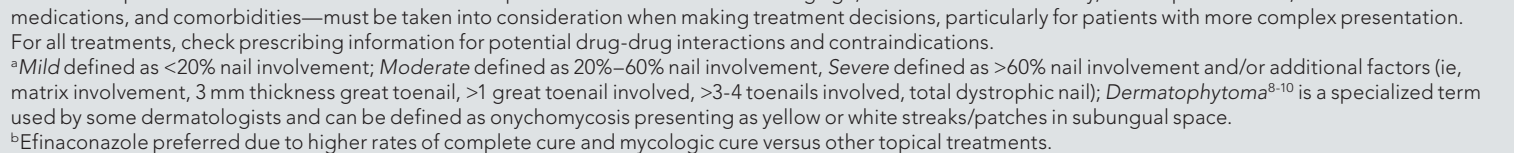

Recommended Medications

(1)

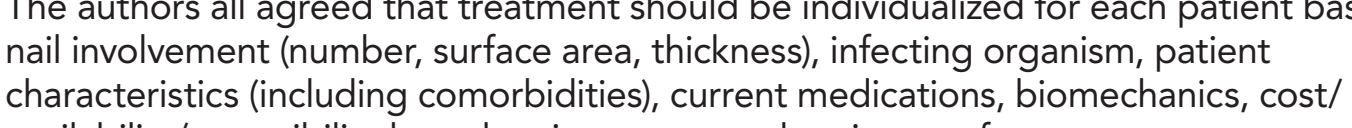
characteristics (including comorbiditites), current medications, biomechanics, cost
availability/accessibility based on insurance, and patient preference

- A decision tree to provide practical guidance on therapeutic recommendations in
onyychomycosis treatment developed by the authors is shown in Figure 2

- Therapeutic recommendations by drug are also detailed in Figure 3

- Among oral medications, terbinafine is most commonly used as first-line treatment,
followed by fluconazole

- Among topical products, efinaconazolo is ideal as firstline medication in pediatric patients,
patients with less severere disease, and those with dermatophyytomas

- A topical medication was also recommended for use in combination with terbinafine or
fluconazole and can be considered as maintenance therapy to prevent relapse

- To improve outcomes, concurrent tinea pedis should be treated in all patients receiving
topical therapy for onychomycosis
- It is important to manage patient expectations when treating onychomycosis: optimal
results can take over a year and clinical cure/normal nail appearance may not be possible

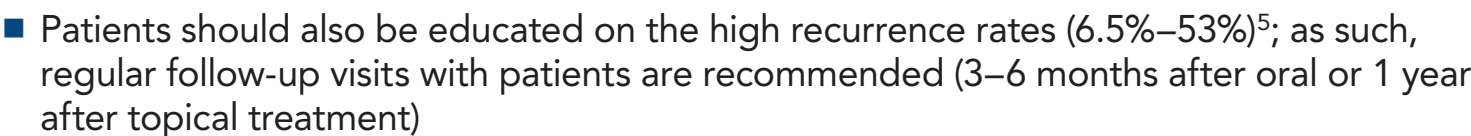
A physical handout (Figure 4) should also be provided to patients, explaining follow-up care/maintenance and highlighting that long-term treatment is more than just
pharmacologic leg personal care, footwear selection/care, land

FIGURE 3. Therapeutic Recommendations by Drug

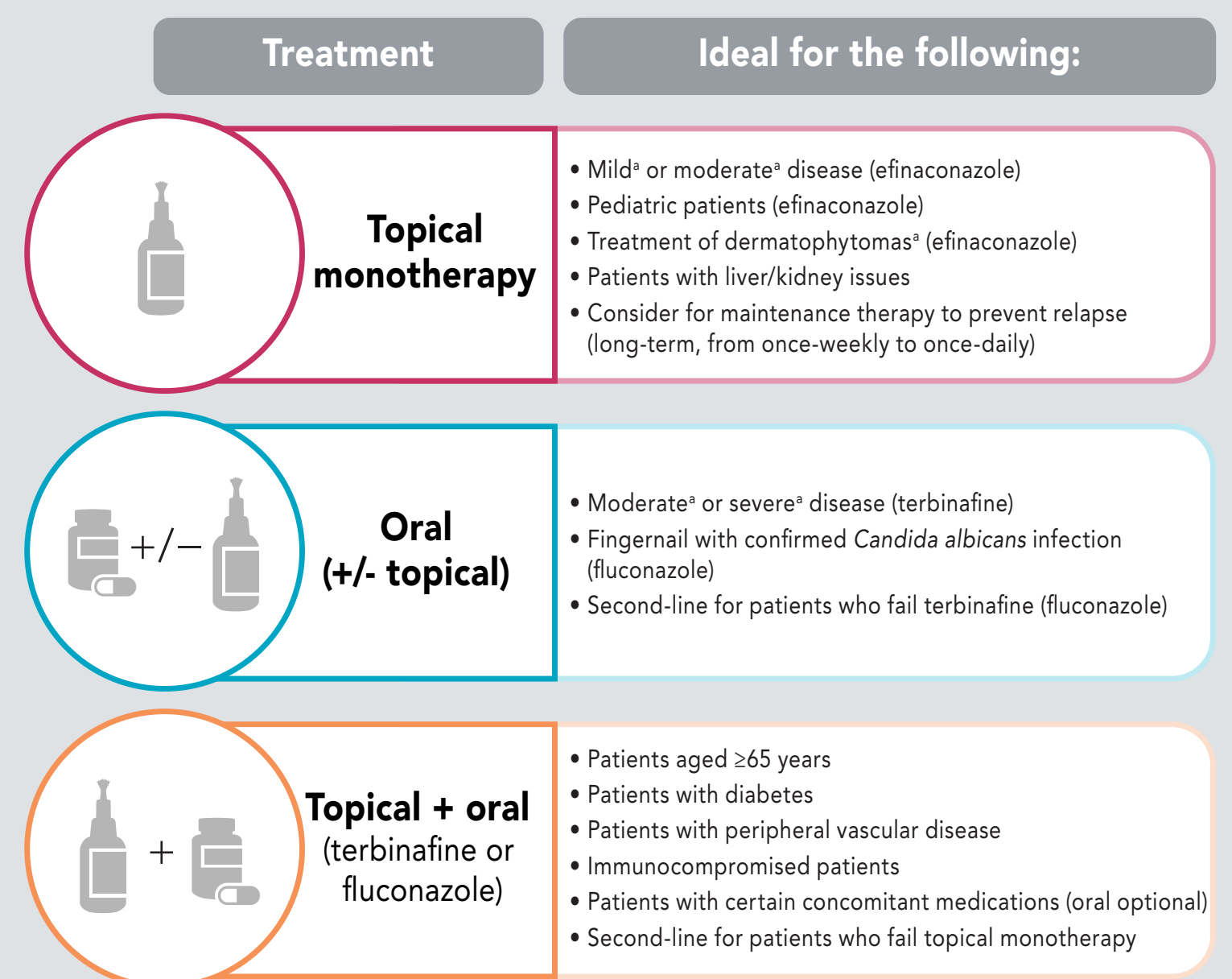

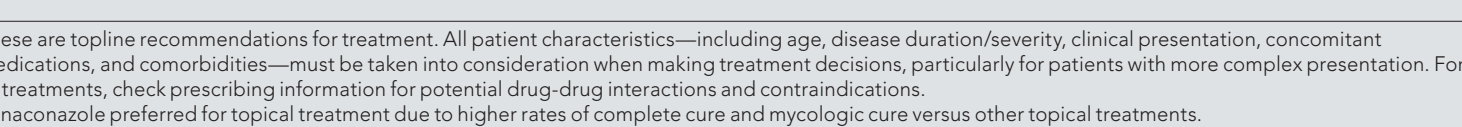

CONCLUSIONS

These therapeutic recommendations, based on new clinical data, provide healthcare practitioners in the diagnosis and treatment of toenail

Onychomycosis should be assessed clinically and confirmed with
microscopy, histology, and/or culture

- Terbinafine is the primary choice for oral treatment and efinaconazole

Efinaconazole can be used for maintenance to prevent recurrence - For optimal outcomes, patients should be counseled regarding treatment
expectations as well as follow-up care and maintenance post-treatment
FIGURE 4. Patient Education Handout

What to know about fungal nail infections

- Noenails grow slowly, so improverements could take $1-2$-2 years to be noticeable - Even after the fungus is sone, the affected nail(s) may never look completetely normal Use treatment(s) recommended by your doctor and
follow the steps below to help prevent new infections:

\section{Personal Care and Laundry}

- Keep nails short and clean

- Only visita alicensed manicurist/pedicurist, bring your own
tools and clean them.

- Don't pick your toenails or scratch your feet with fingernals

- Don't use the same clippersffiles used on abnormal
nails on normal nails.

- Won's share personal nail care instruments, soap, or towels.

- Dryfeet thoroughly after washing

- Wash towels, socks, and clothes after every use. Socks and other
contaminated clothing/towels should be washed 1 at $140^{\circ}$ ( $\left(60^{\circ} \mathrm{C}\right)$.

Footwear

- Wear properly sized shoes with adequate toe boxes. Avoid narrow-toed
shooes or high heels. Avoidnon-breathable athletic shoes.

- Don'twalk barefootin public facilities such as pools,s spas,

- When trying on new shoess, always wear socks.
- Use antifungal spray or powder in your shoes and//r a UV Shoe

- Wear moisture-wicking socks or copper or silver antimicrobial socks

- Replace athletic shoes after 500 miles of use

When to contact your doctor

- If family/household members have athlete's foot or nail infections,
hhey should seek treatment and take precautions to prevent spread

- 'f you see signs of athlete's' foot or reinfection of the nail
contact your doctor as soon as possible

REFERENCES

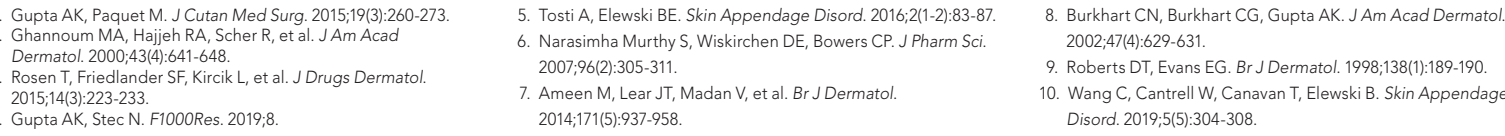

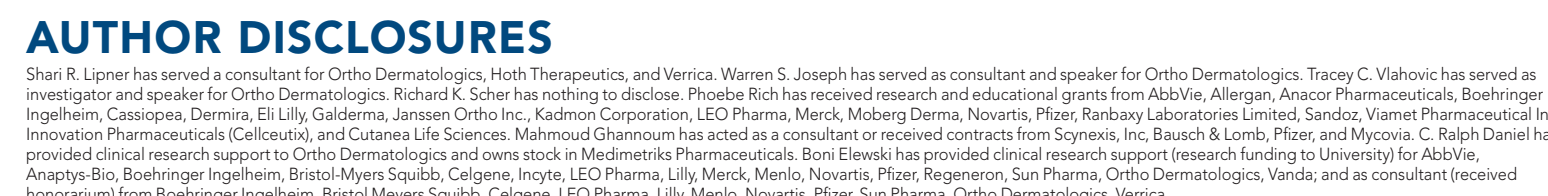

